\title{
Could it be you? The impact of a medico-legal awareness event on junior doctors' understanding of legal issues
}

\author{
Author: Christopher Mitchell
}

Aims

In the wake of the Dr Bawa-Garba case, a heightened level of anxiety existed around medico-legal issues. An evening lecture session for doctors, harnessing local expertise, set out to clarify:

> legal principles surrounding gross clinical negligence manslaughter and the Dr Bawa-Garba case

$>$ the trust's stance with regards to resource-pressured working

$>$ potential legal issues of reflective practice

> what happens during a General Medical Council (GMC) fitness to practise investigation.

\section{Methods}

A lecture evening was arranged to dispel a number of the myths surrounding legal issues underpinning the Dr Bawa-Garba case, and potential vulnerability of junior doctors to similar consequences in the face of systemic failings and increased clinical pressure.

Interactive lectures were given by:

> the assistant director of the University of Southampton's department of clinical law (himself a consultant paediatric surgeon)

> the chief executive, medical director, and director of medical education

$>$ the trust lead on appraisal

$>$ an experienced consultant surgeon who had been through GMC fitness to practise proceedings.

Following the event all attendees were invited to complete online feedback forms, outlining their understanding of the issues covered both pre- and post-event (Fig 1)

\section{Results}

More than 70 clinicians attended the medico-legal issues evening; feedback was obtained from 16 of these.

Following the medico-legal issues evening, attendees reported a significant increase in understanding of principles covered (see Fig 2). The greatest increase - of $63 \%$ - was seen in fitness to practise investigations, followed by medical law underlying gross clinical

Author: Royal College of Physicians chief registrar, University Hospital Southampton NHS Foundation Trust, UK
Questions: $($ Response Options = Strongly Disagree $/$ Disagree $/$ Neutral / Agree / Strongly Agree)

1. I understood the principles of medical law behind gross clinical negligence manslaughter charges (pre- and post-event).

2. I understood the principles and potential issues surrounding reflective practise (pre- and post-event).

3. I understood University Hospital Southampton standpoint with regards to resourcepressured work (pre- and post-event)

4. I understood what happens in a fitness to practise investigation (pre- and post-event).

Fig 1. Survey questions.

negligence manslaughter at $56 \%$, trust stance with regards to resource-pressured work at $31 \%$, and reflective practise potential issues at $19 \%$.

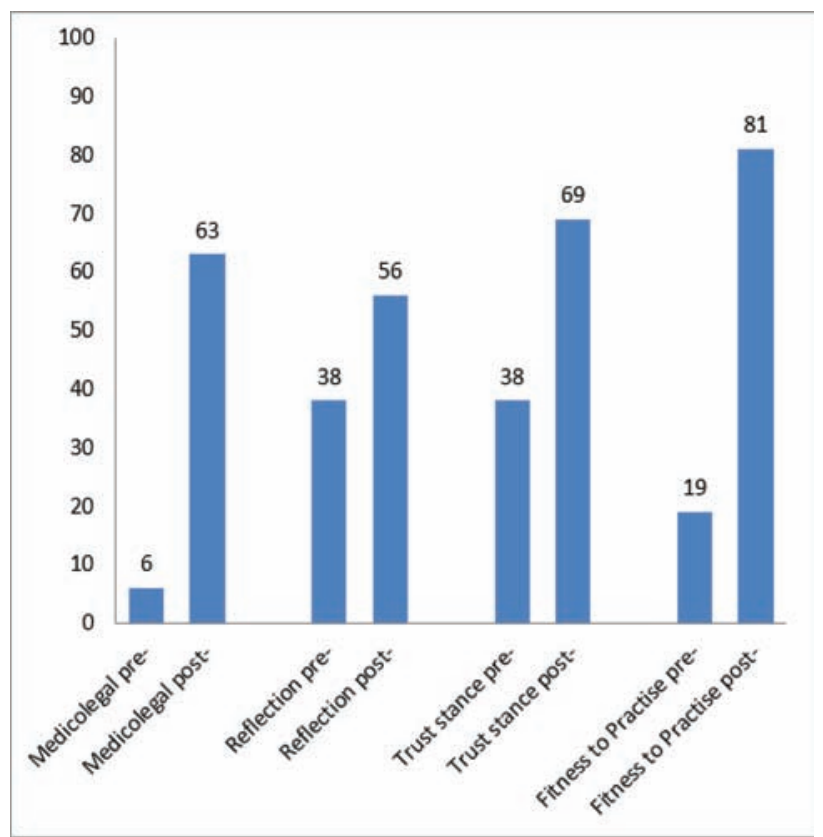

Fig 2. Survey results pre- and post-event. Percentage of attendees who felt they agreed or strongly agreed that they understood the following concepts pre- and post-lecture event: (NB Data subject to change as further feedback is obtained and incorporated into results) 


\section{Conclusion}

Although higher medical and surgical training programmes develop the clinical skills of doctors, little time is dedicated to the processes which result if an adverse event occurs. This teaching session addressed a significant need in the clinician cohort, resulting in significantly increased understanding of principles surrounding medical law, GMC proceedings and reflective practise. A secondary advantage was the increase in profile of the Royal College of Physicians chief registrar role through attendance of colleagues, senior trust managers and senior deanery staff.
Similar sessions should be held in trusts across the country, making use of local expertise to increase clinicians' understanding of these important issues, and demonstrate local support for the invaluable role junior doctors perform.

\section{Conflict of interest statement}

None. 